\title{
Isolated Conjunctival Inflammation Suggestive of IgG4-Related Disease
}

\author{
Hassan A. Aziz ${ }^{a}$ Alexandra Villa-Forte ${ }^{b}$ Thomas P. Plesec ${ }^{c}$ Arun D. Singh ${ }^{a}$ \\ ${ }^{\mathrm{a}}$ Department of Ophthalmic Oncology, Cole Eye Institute, and Departments of ${ }^{\mathrm{b}}$ Rheumatologic and Immunologic \\ Disease and ${ }^{\mathrm{C}}$ Anatomic Pathology, Cleveland Clinic Foundation, Cleveland, Ohio, USA
}

\author{
Key Words \\ Clinical immunopathology · Inflammation · IgG4-related \\ disease
}

\begin{abstract}
Purpose: In this interventional case report, we present the correlation between an isolated conjunctival inflammatory lesion and IgG4-related disease. Results: A 29-year-old female presented with a solitary conjunctival mass of the right eye. The rest of the ocular examination including orbital assessment was negative. Excisional biopsy was suggestive of IgG4-related disease. The patient was treated with oral steroids and remained disease free at 18 months of follow-up. Conclusions: Isolated conjunctival inflammation without orbital involvement can be considered within the spectrum of IgG4-related disease.

(c) 2015 S. Karger AG, Basel

\section{Introduction}

In 2001, Hamano et al. [1] described a new clinical entity called IgG4-related pancreatitis. Soon afterwards, this was recognized to be a systemic disease and was renamed 'IgG4-related disease'. It usually presents as a mass lesion in the pancreas, hepatobiliary tract, orbit and adnexa, lymph nodes, gastrointestinal tract, retroperitoneum and salivary gland amongst other reported sites.
\end{abstract}

\section{KARGER}

E-Mail karger@karger.com

www.karger.com/oop
The orbital and ocular adnexal manifestations of IgG4-related disease is well documented [2-5]. The lacrimal gland, orbit, extraocular muscles, trigeminal nerve branch and periorbital fat are commonly involved. Interestingly, the conjunctiva was consistently noted to be spared in this disease.

Recent case reports described conjunctival involvement in patients with IgG4-related disease [6-8]. Here, we describe an isolated inflammatory conjunctival lesion without orbital involvement that was suggestive of IgG4related disease.

\section{Case Report}

A 29-year-old female was referred to the Ophthalmic Oncology Service at the Cole Eye Institute to rule out conjunctival lymphoma. The patient had a history of an enlarging right bulbar conjunctival lesion for 2 months that was biopsied at an outside institution 1 month prior to referral. The pathology report revealed an inflamed squamous epithelium with an increased number of eosinophils. She had been treated with prednisolone drops every $6 \mathrm{~h}$ in the affected eye with questionable response.

On presentation, her visual acuity was $20 / 20$ in both eyes and the intraocular pressures were 19 and $18 \mathrm{~mm} \mathrm{Hg}$ in the right and left eye, respectively. Ophthalmic examination was essentially normal except for an injected nodular mass measuring $5 \times 5 \times$ $3 \mathrm{~mm}$ located superonasally on the bulbar conjunctiva of the right eye. The lesion had fine multinodular conjunctival thickening along its base (fig. 1a). The patient was free of systemic signs or symptoms and reported no relevant medical or family history. The differential diagnosis included lymphoma, nonspecific or-

Arun D. Singh, MD

Department of Ophthalmic Oncology, Cole Eye Institute 2022 E 105th St

Cleveland, $\mathrm{OH} 44106$ (USA)

E-Mail singha@ccf.org 

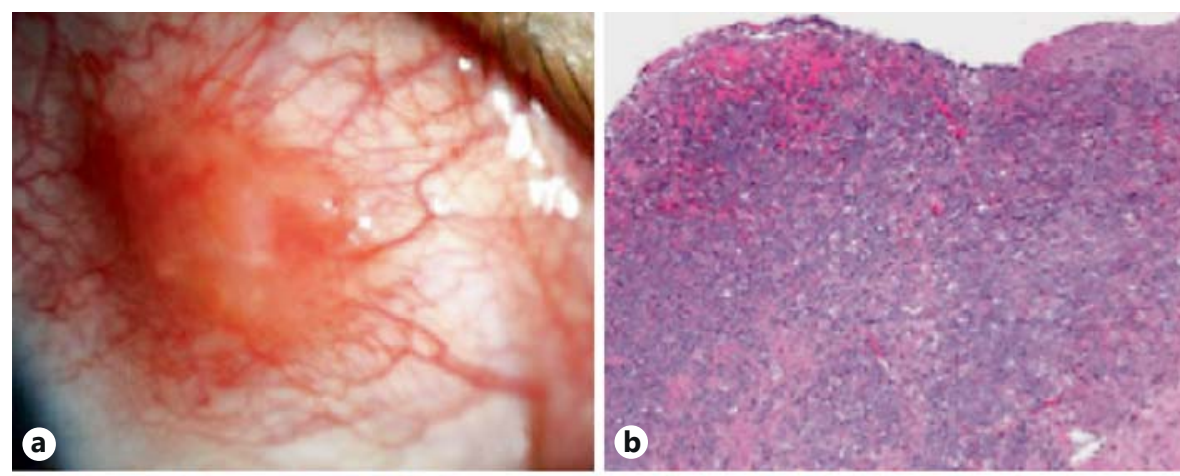

Fig. 1. a Slit-lamp photograph of the right bulbar conjunctiva revealing a multinodular injected cystic mass. b Hematoxylinand-eosin-stained section showing a dense lymphoplasmacytic infiltrate with ulceration. $\times 100$. c IgG immunostaining highlights numerous plasma cells. $\times 200$. d IgG4 immunostaining in the same area highlights up to 101 plasma cells/HPF. The IgG4:IgG ratio is $28 \%$ ( $40 \times$ field was counted $-0.2375 \mathrm{~mm}^{2}$ for IgG4 per HPF). Olympus BX41 microscope. $\times 200$.
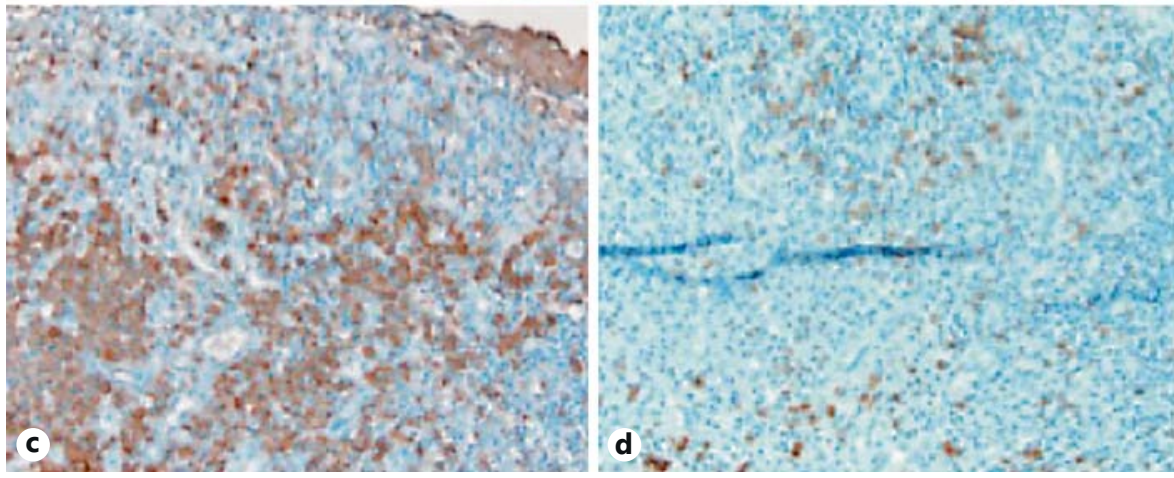

bital inflammation, IgG4-related disease or, less likely, infectious granuloma.

An excisional biopsy was performed, and the pathology revealed ulcerated conjunctiva with fibrosis, numerous plasma cells and 101 IgG4-positive plasma cells in the most affected high-power field (HPF). The IgG4:IgG ratio was 28\% (101/361; fig. 1b-d). Based on the clinical examination and pathology report, the patient was diagnosed with conjunctival inflammation suggestive of IgG4-related disease. Systemic workup by a rheumatologist, including CMP, urinalysis, ESR (4 mm/h, reference range $0-15)$, CRP $(0.1 \mathrm{mg} / \mathrm{dl}$, reference range $0-1.0)$, TSH $(0.764 \mu \mathrm{U} / \mathrm{ml}$, reference range 0.4-5.5), ANCA (negative), and syphilis IgG ( $<0.2 \mathrm{AI}$, reference range $<0.9$ ), was within normal limits, excluding obvious underlying systemic disease. In addition, CT scan of the chest and MRI of the orbit were within normal limits. The patient was started on oral prednisone ( $40 \mathrm{mg}$ ) and was slowly tapered down over the next 6 weeks. On 18 months of follow-up, the patient continues to be symptom free without evidence of systemic disease or ocular recurrence.

\section{Discussion}

Our case describes an isolated conjunctival lesion suggestive of IgG4-related disease in the absence of associated orbital inflammation. While the lacrimal gland is the most common ophthalmic manifestation in IgG4-related disease, the conjunctiva was initially believed to be spared [4]. Recent case reports have shown conjunctival involve-
Table 1. Diagnostic criteria for IgG4-related disease [9]

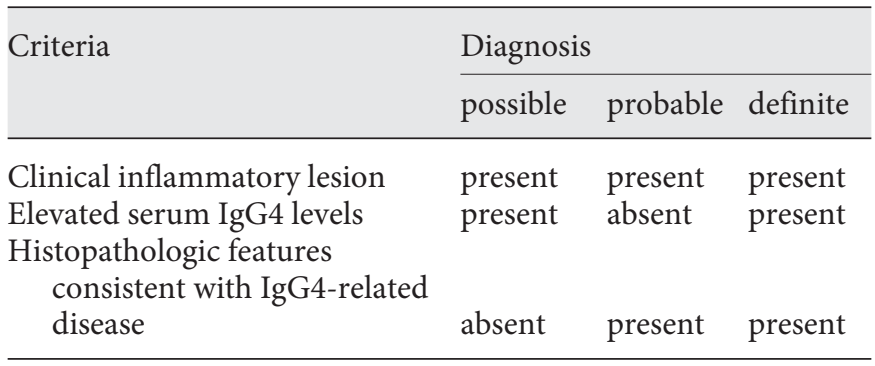

ment in IgG4-related disease with adnexal involvement $[6,8]$ or in isolation [7].

The diagnostic criteria for IgG4-related disease are constantly evolving, as our understanding of this newly described entity is growing. Umehara et al. [9] outlined comprehensive diagnostic criteria for IgG4-related disease that included (1) clinical inflammatory lesion, (2) elevated serum IgG4 levels, and (3) histopathological features consistent with IgG4-related disease (table 1). They concluded that, if all three criteria are present, then a definite diagnosis of IgG4-related disease could be made. Since serum IgG4 levels can be normal in up to $40 \%$ of patients with IgG4-related disease [10], histopathological features are essential in establishing the diagnosis, and 
they include dense lymphocytic infiltrate, fibrosis (at least focally storiform) and obliterative phlebitis [11]. Moreover, a consensus pathology paper showed that histopathological features and clinicopathological correlation are more important for the diagnosis of IgG4-related disease than the suggested cutoffs of $>10$ IgG4 plasma cells/ HPF and an IgG4:IgG ratio of $>40 \%$ [12].

Our patient presented with an isolated inflammatory conjunctival lesion associated with fibrosis (not storiform) and an increase in IgG4 plasma cells (101 cells/ HPF) on pathology. Her serum IgG4 level was not measured prior to treatment and, on pathology, the IgG4:IgG ratio was $28 \%(<40 \%)$ with no clear evidence of phlebitis. The constellation of these findings was suggestive of IgG4-related disease in the absence of systemic or orbital manifestations. Matsuo et al. [13] reported a higher incidence of systemic manifestations when IgG4 involved the ocular adnexa. Thus, the patient continues to be closely monitored for local and systemic recurrence.
Conjunctival inflammation of unknown cause should be considered within the spectrum of ocular and adnexal manifestations of IgG4-related disease. Because of its systemic implications, ophthalmologists should be aware of this diagnosis that could be easily overlooked as idiopathic inflammation.

\section{Statement of Ethics}

The study complied with the guidelines for human studies and animal welfare regulations. The subject gave informed consent, and the study protocol was approved by the institute's committee on human research.

\section{Disclosure Statement}

The authors have no conflicts of interest to declare.

\section{References}

1 Hamano $\mathrm{H}$, et al: High serum IgG4 concentrations in patients with sclerosing pancreatitis. N Engl J Med 2001;344:732-738.

-2 Sato Y, et al: Ocular adnexal IgG4-related disease has uniform clinicopathology. Pathol Int 2008;58:465-470.

3 Kubota T, et al: Ocular adnexal IgG4-related lymphoplasmacytic infiltrative disorder. Arch Ophthalmol 2010;128:577-584.

4 Sogabe Y, et al: Location and frequency of lesions in patients with IgG4-related ophthalmic diseases. Graefes Arch Clin Exp Ophthalmol 2014;252:531-538.

5 Pasquali $\mathrm{T}$, et al: Orbital inflammation in IgG4-related sclerosing disease. Orbit 2011; $30: 258-260$
6 Paulus YM, et al: IgG4-positive sclerosing orbital inflammation involving the conjunctiva: a case report. Ocul Immunol Inflamm 2012; 20:375-377

7 Philippakis E, et al: IgG4-related disease masquerading as recurrent scleritis and chronic conjunctivitis. Ocul Immunol Inflamm 2015; 23:168-172.

8 da Fonseca FL, et al: Unilateral eyelid mass as an unusual presentation of ocular adnexal IgG4-related inflammation. Cornea 2013;32: 517-519.

-9 Umehara H, et al: Comprehensive diagnostic criteria for IgG4-related disease (IgG4-RD), 2011. Mod Rheumatol 2012;22:21-30.
10 Carruthers MN, et al: The diagnostic utility of serum IgG4 concentrations in IgG4-related disease. Ann Rheum Dis 2015;74:14-18.

-11 McNab AA, McKelvie P: IgG4-related ophthalmic disease. Part I: background and pathology. Ophthal Plast Reconstr Surg 2015;31: 83-88.

12 Deshpande V, et al: Consensus statement on the pathology of IgG4-related disease. Mod Pathol 2012;25:1181-1192.

13 Matsuo T, et al: Immunoglobulin G4 (IgG4) positive or -negative ocular adnexal benign lymphoid lesions in relation to systemic involvement. J Clin Exp Hematop 2010;50:129142
Isolated Conjunctival Inflammation Suggestive of IgG4-Related Disease 